\title{
Instrumental setup for polarized neutron scattering experiments
}

\author{
W. Schmidt ${ }^{\mathrm{a})}$ and M. Ohl \\ Forschungszentrum Jülich, Institut für Festkörperforschung, 52425 Jülich, Germany \\ H. Ruppert \\ University of Applied Science Darmstadt, 64295 Darmstadt, Germany \\ U. Buchenau \\ Forschungszentrum Jülich, Institut für Festkörperforschung, 52425 Jülich, Germany
}

(Presented on 13 November 2002)

\begin{abstract}
Neutron scattering with polarization analysis is a powerful tool to determine magnetic structures and excitations. A common setup is to mount the sample at the center of a Helmholtz-type coil which can provide a magnetic field of any direction at the sample position and also a guide field along the neutron flight paths around the sample. We have extended the standard setup to a combination of a large vacuum tank surrounded by the electrical coils. The vacuum tank eliminates the air scattering, and we can use the polarization analysis down to the lowest accessible momentum transfers. The coils themselves also show some appealing features: In contrast to the classic (symmetric) coil distribution, we use an asymmetric setup which gives the advantage of a larger scattering window. Due to a more sophisticated current distribution, this modified Helmholtz coil does not need to be rotated for different scattering conditions. The whole setup is currently being constructed at the IN12 instrument, a three-axis spectrometer for cold neutrons, operated by FZ Jülich in collaboration with CEA Grenoble as a CRG-B instrument at the Institut Laue Langevin in Grenoble. (c) 2003 American Institute of Physics. [DOI: 10.1063/1.1558188]
\end{abstract}

\section{INTRODUCTION}

In recent years there has been a rather high demand for inelastic neutron scattering experiments at low momentum transfers. There are examples both from soft-matter physics and magnetism. ${ }^{1,2}$ Due to the low scattering angles in these measurements, the air scattering around the sample causes huge experimental problems. This can be mastered by using a large vacuum tank around the sample as shown in Fig. 1. This technique had been in use at the Institut Laue Langevin (ILL) for quite some time. In the meantime, we have constructed a tank which also can be mounted together with a Helmholtz-type setup of electrical coils. This provides an appealing feature: The tank can now also be used with polarized neutrons which give a much better flexibility in addressing problems in magnetism and also soft-matter physics, like distinguishing between coherent and incoherent scattering. The coils themselves show some interesting features which will be explained in detail.

\section{DESIGN OF THE COILS}

The first part of the setup consists of two ring coils of radius $a$ arranged horizontally in equal distance $h=a / 2$ above and below the sample position to provide a homogenous vertical field in the scattering plane. The second part consists of three window coils where each of them covers a certain section along the two ring coils connected by two straight vertical parts which in total form the three pillars of the whole arrangement. These coils will provide a horizontal field at the sample position. A schematic view of this setup is

a) Also at: Institut Laue Langevin, BP 156, 38042 Grenoble, France; electronic mail: schmidt@ill.fr shown in Fig. 2. For the calculations, the coils are idealized as if they were built from a single wire so that standard methods from textbook magnetostatics can be used.

The window coils are numbered from one to three while the angular size of coil No. 1 is a free parameter defined as $4 \xi$. Coil Nos. 2 and 3 are set identical; with the previous definition, their angular sizes are both $\pi-2 \xi$. In contrast to the classic (symmetric) setup where each of the windows covers $120^{\circ}(\xi=\pi / 6)$, this modification allows one larger coil and therefore a larger scattering window for the outgoing neutron beam. For the construction, we finally chose $150^{\circ}$ for coil No. $1(\xi=5 \pi / 24)$. Considering the thickness of the pillars, this allows a scattering window of at least $120^{\circ}$, sufficient for most applications.

The $x$ axis of the coordinate system is defined by the direction of the incoming neutron beam. The angle between the center of coil No. 1 and the $x$ axis is called $\alpha_{0}$. With this single angle, the orientation of all three coils is defined. Taking into account the given angular sizes, the centers of coil Nos. 2 and 3 are set as $\alpha_{0} \mp(\xi+\pi / 2)$.

With the electrical currents $I_{1}, I_{2}, I_{3}$, the number of turns $N$ and the magnetic permeability $\mu_{0}$, the magnetic field of the window coils at the sample position calculated from the idealized window coils is given by

$$
\begin{aligned}
B_{x}= & \frac{9 \mu_{0} N}{10 \sqrt{5} \pi h}\left[I_{1} \cos \alpha_{0} \sin 2 \xi+I_{2} \sin \left(\alpha_{0}-\xi\right) \cos \xi\right. \\
& \left.-I_{3} \sin \left(\alpha_{0}+\xi\right) \cos \xi\right], \\
B_{y}= & \frac{9 \mu_{0} N}{10 \sqrt{5} \pi h}\left[I_{1} \sin \alpha_{0} \sin 2 \xi-I_{2} \cos \left(\alpha_{0}-\xi\right) \cos \xi\right. \\
& \left.+I_{3} \cos \left(\alpha_{0}+\xi\right) \cos \xi\right] .
\end{aligned}
$$




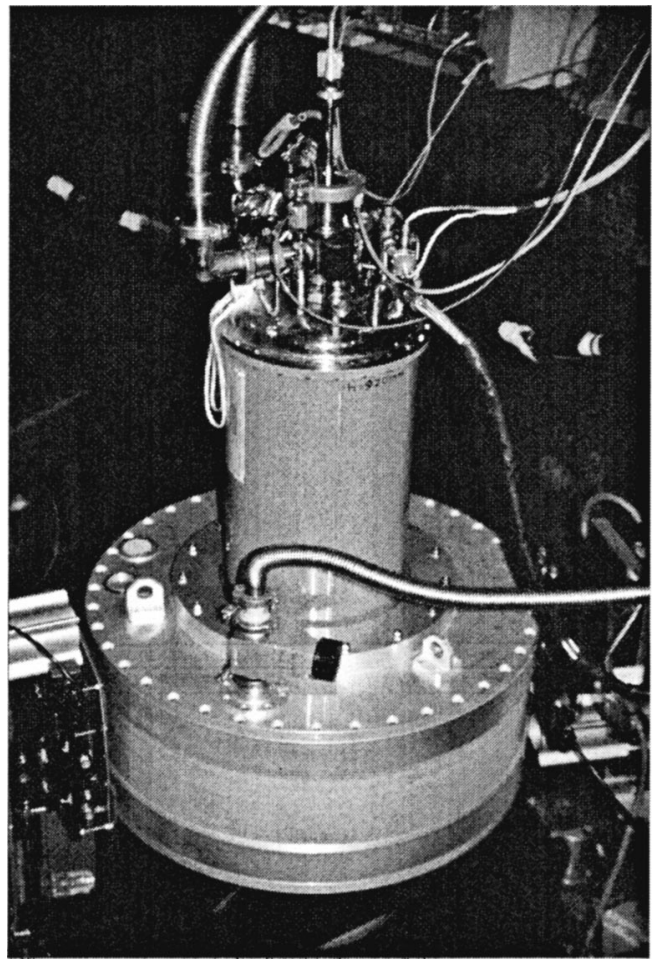

FIG. 1. The vacuum tank on the three-axis spectrometer IN12 fitted with a standard orange cryostat.

With the condition that we need a field of a certain size and direction, we set

$$
B_{x}=B_{0} \cos \beta, \quad B_{y}=B_{0} \sin \beta,
$$

and with the abbreviations

$$
I_{0}=\frac{5 \sqrt{5} \pi h B_{0}}{9 \sqrt{3} \mu_{0} N \sin \xi \cos ^{2} \xi}, \quad \psi=\alpha_{0}-\beta,
$$

we get $I_{2}$ and $I_{3}$ independent of $I_{1}$ from Eq. (1):

$$
I_{2 / 3}=I_{1}-\sqrt{3} I_{0} \cos (\psi \pm \xi) \text {. }
$$

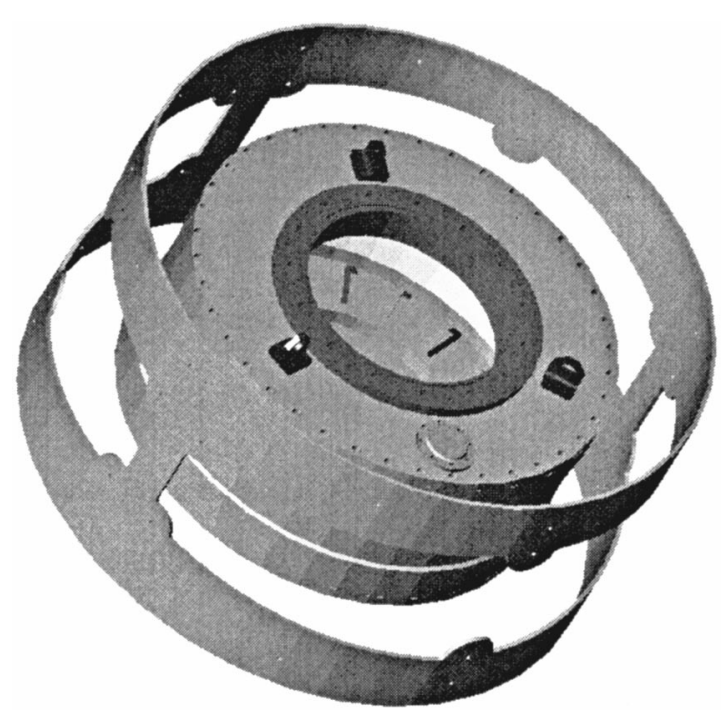

FIG. 2. A schematic drawing of the outer frame for the coils around the vacuum tank. The electrical wiring is omitted for clarity.
Finally, $I_{1}$ can be determined by an optimization of the total electrical power of the three coils, given by the currents and their resistances $R_{1}$ for the larger coil and $R_{2}$ for the two smaller coils:

$$
P=R_{1} I_{1}^{2}+R_{2} I_{2}^{2}+R_{2} I_{3}^{2} .
$$

Using Eq. (4) and solving $\partial P / \partial I_{1}=0$, we get $I_{1}$ as

$$
I_{1}=\frac{2 R_{2}}{R_{1}+2 R_{2}} \sqrt{3} I_{0} \cos \psi \cos \xi
$$

With this solution, we can give the complete and well-known solution for the symmetric case with $R_{1}=R_{2}, \xi=\pi / 6$, $I_{0, \text { sym }}=I_{0}(\xi=\pi / 6)$ :

$$
I_{1, \text { sym }}=I_{0, \text { sym }} \cos \psi, \quad I_{2 / 3, \text { sym }}=I_{0, \text { sym }} \cos (\psi \mp 2 \pi / 3) .
$$

Important parameters for the electrical design of the coils are the amplitudes of the currents. From Eq. (7), we get the same value of $I_{0, \text { sym }}$ for all coils. To calculate the values for the asymmetric case, we rewrite $I_{2}$ and $I_{3}$ in a form like Eq. (7), introducing an amplitude factor $A$ and an offset angle $\delta$ :

$$
I_{2 / 3}=A I_{0} \cos (\psi \mp 2 \pi / 3 \mp \delta),
$$

and we can write the current amplitudes

$$
I_{1, \text { max }}=\frac{2 R_{2}}{R_{1}+2 R_{2}} \sqrt{3} I_{0} \cos \xi, \quad I_{2 / 3, \text { max }}=A I_{0},
$$

while the parameters $A$ and $\delta$ can be easily calculated using Eqs. (4) and (6).

A further optimization can now be achieved by choosing a convenient ratio for $R_{2} / R_{1}$. Technically, this can be realized by choosing appropriate cross sections of the used wire. In this respect, an important constraint is the maximum voltage of the power supplies for the electrical currents. Therefore, the amplitude of these currents should match the maximum voltage:

$$
U_{\max }=R_{1} I_{1, \max }=R_{2} I_{2 / 3, \max } .
$$

Using Eq. (9) and the calculation for $A$ and $\delta$, we get

$$
2 R_{2} / R_{1}=\sqrt{3} \cot \xi-1, \quad A=2 \sin \xi, \quad \delta=0,
$$

and also, the final results for the currents:

$$
\begin{aligned}
& I_{1}=2 \sin (\pi / 3-\xi) \cdot I_{0} \cos \psi, \\
& I_{2 / 3}=2 \sin \xi \cdot I_{0} \cos (\psi \mp 2 \pi / 3) .
\end{aligned}
$$

An important result is the fact that the voltage condition implicitly delivers $\delta=0$ which allows one to implement the asymmetric coils in the same way as the symmetric coils except with different amplitude factors. For the considered technical realization $(\xi=5 \pi / 24)$, these factors with respect to the symmetric case are then 0.749 for $I_{1}$ and 1.192 for $I_{2 / 3}$. The corresponding resistance ratio is $R_{2} / R_{1}=0.629$. A factor of about 0.78 will already be provided from geometry (longer wire needed for the larger coil), the rest can be adjusted by choosing a slightly smaller wire cross section for coil No. 1. 


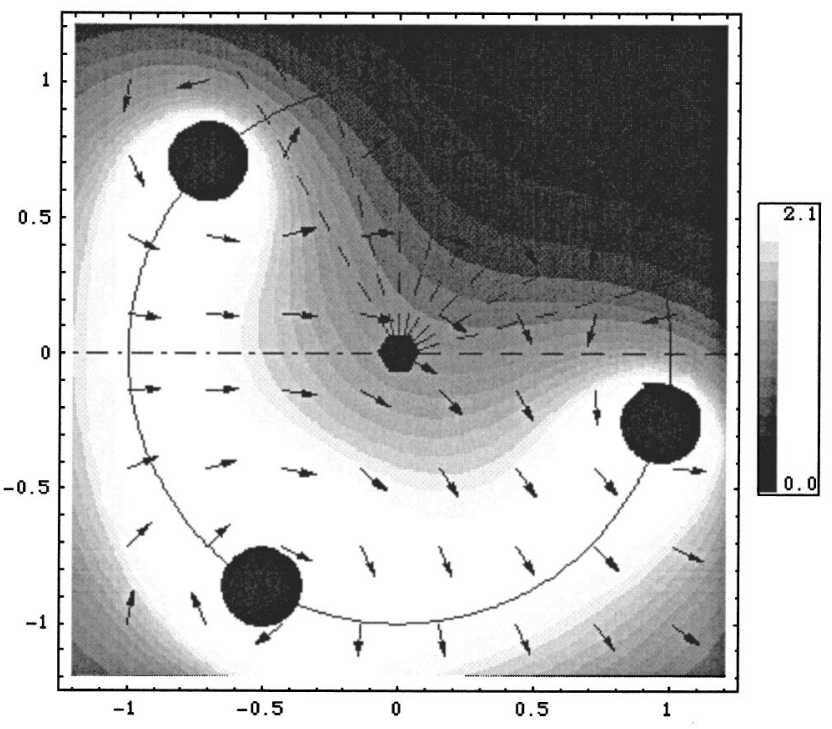

FIG. 3. Contours of the horizontal magnetic field strength in the scattering plane. The radius of the ring coils and also the field at the center have been normalized to one. Also shown is the large circle of the coils with the three pillars. The hexagon at the center indicates the sample position, the arrows show the direction of the magnetic field. The dotted-dashed line stands for the incoming beam while the dashed lines through the larger window coil symbolize the range of the scattered outgoing beam.

\section{GUIDE FIELD DISCUSSION}

In addition to generating a magnetic field at the sample position, the coils also must provide a guide field for the incoming and scattered neutron beam throughout the whole interior of the setup, at least up to the outer borders of the vacuum tank. Outside the tank, external guide fields may take over.

For a vertical guide field (generated by the two ring coils), the guide field issue causes no problems. At the border of the vacuum tank (a few centimeters less than the ring coil radius), the field will still be more than $50 \%$ of the field at the center.

For the horizontal case, a more detailed consideration is necessary. Depending on the orientation of the coils and the direction of the field at the center, the electric current through one of the window coils may be very low or even vanish and therefore cause a rapid decrease of the magnetic field in the range of this specific coil. Equations (7) and (12) easily give $I_{1}=0$ when $\cos \psi$ vanishes. This may be avoided by turning the whole setup, i.e., changing $\alpha_{0}$ and also $\psi$ [Eq. (3)].

In the asymmetric case, however, we want to take advantage of the larger window of coil No. 1 for the outgoing beam and therefore prefer to keep the whole setup fixed. Figure 3 shows a contour plot of the magnetic field strength for the optimum orientation with respect to the scattering conditions $\left(\alpha_{0}=\pi / 3\right)$ as well as the extreme case $I_{1}=0$ for $\beta=-\pi / 6$. To counteract the decline of the field toward coil No. 1, we have two other possibilities besides turning the coils.

The currents in all three window coils are increased by the same value. This will superpose an additional guide field

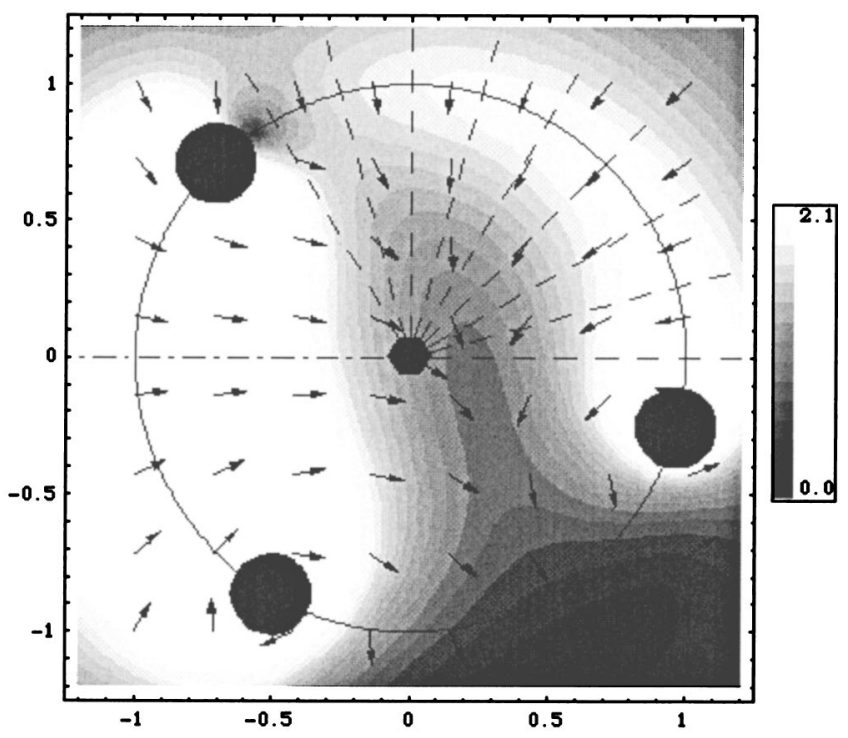

FIG. 4. The same setup as shown in Fig. 3 with additional currents both through the ring and window coils to provide a sufficiently high guide field for the scattered beam.

toward the rim of the coils but leave the field at the sample position unchanged. This can easily be proven by Eq. (1): Setting $I_{1}=I_{2}=I_{3}$ yields $B_{x}=B_{y}=0$, independent of the other parameters.

Also the ring coils can be used to impose an additional horizontal guide field. We just feed these coils with currents in opposite directions. Then, in the scattering plane, the vertical field vanishes and instead a radial field is created there. Again, the field at the sample position remains unchanged. If a vertical field is needed in addition, the upper and lower coil are fed with different currents $I_{\text {up }}$ and $I_{\mathrm{dn}}$. Now ( $I_{\text {up }}$ $\left.+I_{\mathrm{dn}}\right) / 2$ contributes to the vertical field while $\left(I_{\text {up }}-I_{\mathrm{dn}}\right) / 2$ creates a horizontal guide field.

Figure 4 shows an example to counteract the decline of the guide field with these methods. Both for the ring coil and the window coils, we have chosen an additional current of $60 \%$ of $I_{0}$ as defined in Eq. (3).

\section{CONCLUSION}

The combination of a vacuum tank with a Helmholtz coil will create interesting possibilities for polarized neutron experiments. We also demonstrated that the asymmetric coil design is technically feasible and shows clear advantages to the classical setup. The technical construction of the coils is currently being done. We expect the whole setup to be available at IN12 and also other instruments of ILL beginning in 2003.

\section{ACKNOWLEDGMENT}

This work has been funded by the German Research Ministry BMBF under Contract No. 05-300-CJB-6.

${ }^{1}$ A. Schreyer, H. Tartakowskaya, A. Wildes, W. Schmidt, R. Erwin, S. H. Lee, C. F. Majkrzak, M. Hong, and J. Kwo, Proceedings SCNS Workshop, ILL, Grenoble, France (2002).

${ }^{2}$ W. Schmidt, M. Ohl, and U. Buchenau, Appl. Phys. A: Mater. Sci. Process. 74, 5537 (2002) 